\title{
Full Blood Count Estimation Using Abbott Cell-Dyn Ruby 5-Parts in Comparison with Boule Medonic M-Series 3-Parts Differential Haematology Analyser
}

\author{
Bolarinde Joseph Lawall,2*, Solomon Umukoro², Nuredin Ibrahim Mohammed ${ }^{3}$, Mustapha \\ Dibbasey², Ida Jallow Manneh², Mamudou Dahaba², Chibuike Nnadozie Okpara ${ }^{4}$, Karen For- \\ rest $^{4}$, Annette Erhart ${ }^{5}$ and Davis Nwakanma ${ }^{2}$ \\ ${ }^{1}$ Clinical Research Department, London School of Hygiene ad Tropical Medicine, The Gambia \\ ${ }^{2}$ Laboratory Services, MRC Unit the Gambia at the London School of Hygiene and Tropical Medicine, The Gambia
}

${ }^{3}$ Data Management and Bioinformatics, MRC Unit the Gambia at the London School of Hygiene and Tropical Medicine, The Gambia

${ }^{4}$ Clinical Services, MRC Unit the Gambia at the London School of Hygiene and Tropical Medicine, The Gambia

${ }^{5}$ Disease Control and Elimination, MRC Unit the Gambia at the London School of Hygiene and Tropical Medicine, The Gambia

*Corresponding author: Bolarinde Joseph Lawal, Ebola Vaccine Trial Laboratory, London School of Hygiene and Tropical Medicine, Kambia District, Sierra Leone. The Gambia.

To Cite This Article: Bolarinde Joseph Lawal, Solomon Umukoro, Nuredin Ibrahim Mohammed, Mustapha Dibbasey, Full Blood Count Estimation Using Abbott Cell-Dyn Ruby 5-Parts in Comparison with Boule Medonic M-Series 3-Parts Differential Haematology Analyser. Am J Biomed Sci \& Res. 2020 - 11(3). AJBSR.MS.ID.001636. DOI: 10.34297/AJBSR.2020.11.001636.

Received: 眥 November 30, 2020; Published: 㠿 December 21, 2020

\begin{abstract}
Full Blood Count (FBC) is a significant laboratory investigation often requested in clinical care and trials; laboratories should have comparable alternative methods for this test to ensure uninterrupted services. This study evaluated to what extent the Abbott CELL-DYN Ruby and Boule Medonic M-series haematology analysers can be used interchangeably. Using 110 leftover EDTA blood samples to test twelve haematological parameters, correlations and bias between the two analysers for each blood parameter were determined by plotting Passing \& Bablok and Bland-Altman's plots respectively. The two analysers were assessed comparable for a test when the bias with the $95 \%$ CI falls within the clinically acceptable limits which had been defined a priori. All the twelve FBC parameters showed strong positive correlations between the measurements from the two analysers. The measurements on both analysers however showed some bias for all the parameters evaluated except Red Blood Cells (RBC), whose bias was $0.00 \times 10-12 / \mathrm{L}(-0.01,0.02)$. The observed biases met the acceptance criteria, except Haematocrit (HCT), Mean Cell Volume (MCV) and Lymphocytes -\% (Lym-\%). Abbott CELL-DYN Ruby and Boule Medonic M-series haematology analysers are comparable for Haemoglobin (HGB), White Blood Cell Count (WBC), RBC, Platelets (PLT), Mean Cell Haemoglobin (MCH), Mean Cell Haemoglobin Concentration (MCHC), Red Cell Distribution Width (RDW), Mean Platelets Volume (MPV) and Lymphocytes -Absolute (Lym-Abs), but not for HCT, MCV, and LYM which will require interpreting results using equipment-specific reference intervals. The results of this study may help laboratories and clinics in selecting comparable methods for FBC. Moreover, the two study analysers have closed sampling facilities which make them safe for handling suspected infectious samples, as contact with personnel is minimal.
\end{abstract}

Keywords: Cell-DYN Ruby; Medonic-M Series; Full Blood Count; Method Comparison

Abbreviations: HGB: Haemoglobin; WBC: White Blood Cell Count; HCT: Haematocrit; RBC: Red Blood Cells Count; PLT: Platelets; MCV: Mean Cell Volume; MCH: Mean Cell Haemoglobin; MCHC: Mean Cell Haemoglobin Concentration; RDW: Red Cell Distribution Width; MPV: Mean Platelets Volume; Lym-Abs: Lymphocytes -Absolute; Lym-\%: Lymphocytes -\%; CD-Ruby: Abbott CELL-DYN Ruby; Medonic-M: Medonic M-series; CLSI: Clinical and Laboratory Standard Institute; MRCG at LSHTM: MRC Unit The Gambia at the London School of Hygiene and Tropical Medicine; LSHTM: London School of Hygiene and Tropical Medicine. 


\section{Introduction}

Full Blood Count (FBC) also referred to as Complete Blood Count (CBC) is an important laboratory investigation required for disease diagnosis, patient management, subject screening and safety testing for vaccine or drugs during clinical trials [1-3]. A recent assessment of all laboratory test requests made from general practice in Oxfordshire, UK, showed FBC as the second most commonly requested blood tests [4]. As a result of its importance in healthcare delivery, it is crucial that Haematology laboratories provide FBC tests consistently. To achieve this, the laboratory must be equipped with alternative options, hence the need to have comparable haematological analysers that can be used interchangeably. The MRCG at LSHTM Clinical laboratory recently acquired the Abbott CELLDYN Ruby (Abbott Laboratories, Diagnostic Division, Abbott Park, IL, USA) 5-parts differential haematology analyser as an addition to the existing one.

As part of quality assurance procedures, and in fulfilment of ISO15189 requirement, the newly acquired CELL-DYN Ruby analyser was evaluated before it was commissioned for use. The evaluation process included comparing the new analyser with the existing Medonic M-series (Medonic-M) analyser (manufactured by Boule Medical, SWEDEN). Clinical laboratories' method comparisons often involve estimating "bias" between the methods in addition to correlation analysis. Bias in this context expresses the closeness of agreement between the two methods [5] and is currently used as one of the most important components of method verification in clinical laboratories when establishing comparability between two methods [6].

As an alternative to estimating limits of agreement, this study assessed the clinical significance of observed bias between the two analysers to determine to what extent the two methods can be used interchangeably.

\section{Materials and Methods}

We followed the Clinical and Laboratories Standard Institute (CLSI) guidelines on Measurement Procedure Comparison and Bias Estimation Using Patient Samples [7] to compare full blood counts using both analysers simultaneously.

\section{Study setting and sampling}

This study was carried out at the IS015189:2012 accredited Clinical Laboratories of the Medical Research Council Unit The Gambia at the London School of Hygiene \& Tropical Medicine (MRCG at LSHTM), as part of the method evaluation prior to commissioning of the CD-Ruby analyser for use in the Haematology laboratory. Anonymised leftover EDTA-anticoagulated clinical blood samples submitted for routine FBC, including both normal and pathological samples, ranging from low to high measurement parameters concentrations, were used for this method comparison. Sample selection criteria were strictly based on the available volume, i.e., samples with insufficient volume to accommodate a minimum of two runs were not included in the study. The samples were collected into Becton-Dickinson vacutainer Ethylene Diamine Tetra Acetic acid (EDTA)-anticoagulated tube (Becton Dickson vacutainer, cat. No. 388010) by a competent phlebotomist. The samples were maintained and transported into the Haematology laboratory within one hour at room temperature. After completing the requested haematological assays on the EDTA samples, residual samples were anonymously analysed simultaneously using both analysers.

\section{Sample analysis}

The samples were analysed using Medonic-M 3-parts haematology analyser as reference method, and CD Ruby 5-parts analyser as the candidate method. Quality controls were run prior to sample analysis to ascertain the functionality of both analysers as required by the IS015189:2012 standard [8].

Medonic-M analyser is a fully automated haematology analyser intended for in vitro examination of human blood samples under well-monitored laboratory condition. The measuring principles of the Medonic-M are based on impedance for cell count and spectrophotometry for Haemoglobin (HGB) [9].

CD-Ruby is an integrated multi-parameter full automated haematology analyser designed for in vitro diagnostic use in clinical laboratories. The instrument measures blood parameters through laser flow cytometry. The analyser uses $632.8 \mathrm{~nm}$ helium-neon laser as the light source and four optical detectors for the scattered light detection; $0^{\circ}-3^{\circ}$ for cell size, $10^{\circ}$ for cells internal complexity, $90^{\circ}$-polarised for lobularity and $90^{\circ}$-depolarised for the specific identification of polynuclear eosinophils (PNE). For the counting of Red Blood Cells (RBCs) the scattered light is measured at $0^{\circ}, 10^{\circ}$, and $90^{\circ}$ and for Platelets (PLTs), at $0^{\circ}$ and $10^{\circ}$. WBCs counts and differentials are established by the light scattered at four different detectors located in the forward $\left(0^{\circ}\right.$ and $\left.10^{\circ}\right)$ and side $\left(90^{\circ}\right.$ and $90^{\circ} \mathrm{D}$ ) angles. The information from these four detector channels is used to construct the five different types of WBCs by a process known as Multi-Angle-Polarised-Scatter-Separation. In the case of sample containing fragile WBCs or resistant RBCs, an alternative mode of FBC analysis known as Nucleated Optical Count (NOC) is used to accurately determine WBCs count. For HGB concentration, a low-energy LED attached to the HGB Flow Cell measures the absorbance of light at $555 \mathrm{~nm}$ and the absorbance is proportional to the HGB concentration of the sample [10].

Prior to analyses, the two analysers were calibrated, and routine maintenance were performed in accordance with the manufacturer's recommendations and internal quality management system. 


\section{Statistical Analysis}

The CLSI recommends a minimum of 40 patient samples to establish bias between two methods [7]. We used 110 samples to improve the confidence in our statistical estimates and to incorporate the effects of unexpected interfering substances.

The data were entered and managed using Microsoft Access $₫$ Version 12.0. Statistical analysis was done using the MedCalc for Windows, version 17.6 [11] and Minitab Version 18.1 [12]. Data were visually inspected for outliers using plots but formal assessment and decision to remove outliers was based on Turkey's method [13].

The CLSI guidelines [7] recommend that clinical laboratories may provide a statement of bias determination through either regression or difference plots; this study presents results from the latter, using Bland-Altman plots [14]. Variability and linear relationship in differences between the two analysers' measurements of each parameter were assessed using scatter plots based on Passing \& Bablok regression $[7,15]$. A correlation was considered strong if the correlation coefficient (R) was 0.6 or more $[16,17]$. Deviation from linear relationship was tested using the Cusum test [18]. We used the Anderson-Darling method [19] to check for normality in the distribution of the differences. For tests whose differences were normally distributed, Bland-Altman's plots were made, the average overall bias for the measured range was simply computed as the mean of all the differences. On the other hand, for the parameters whose differences data followed a skewed distribution, the median of the difference at $95 \% \mathrm{CI}$ interpolation values represented the bias [7]. We established that the two methods can be used interchangeably for a given haematological index if the bias and related 95\% CI fell within the limits of clinical acceptability which we had defined a priori in collaboration with clinicians and in consideration of the total allowable errors [20,21].

\section{Ethics}

The study was approved by the Science Coordinating Committee of MRCG at LSHTM and the Gambia Government/MRCG Joint Ethics Committee.

\section{Results and Discussion}

\section{Results}

We compared the CD-Ruby and Medonic-M for the twelve following haematological parameters: (i) Haemoglobin (HGB) , (ii) White Blood Cells count (WBC), (iii) Haematocrit (HCT), (iv) Red Blood Cells count (RBC), (v) Platelets (PLT), (vi) Mean Cells Volume (MCV), (vii) Mean Cell Haemoglobin (MCH), (viii) Mean Cell Haemoglobin Concentration (MCHC), (ix) Red cells Distribution Width (RDW), (x) Mean Platelets Volume (MPV), (xi) Absolute Lymphocytes [Lym-Abs], and (xii) percentage Lymphocytes [Lym(\%)] (Table 1).

Table 1: Description of the blood samples evaluated by CELL-DYN RUBY and Medonic M-Series Haematology Analysers.

\begin{tabular}{|c|c|c|c|c|c|c|c|}
\hline \multirow{2}{*}{ Parameters Measured } & \multirow{2}{*}{$\begin{array}{c}\text { Sample } \\
\text { SIZE (N) } \\
\end{array}$} & \multicolumn{3}{|c|}{ Medonic M Series } & \multicolumn{3}{|c|}{ Celldyn Ruby } \\
\hline & & Measured Range & Mean & SD & Measured Range & Mean & SD \\
\hline Haemoglobin (HGB) & 107 & $5.60-17.2 \mathrm{~g} / \mathrm{dl}$ & 11.84 & 2.36 & $5.5-18.4 \mathrm{~g} / \mathrm{dl}$ & 12.68 & 2.65 \\
\hline $\begin{array}{l}\text { White Blood Cell Count } \\
\text { (WBC) }\end{array}$ & 106 & $2.8-20.2 \times 10^{9} / \mathrm{L}$ & 6.5 & 5.9 & $2.7-20.7 \times 10^{9} / \mathrm{L}$ & 6.6 & 2.7 \\
\hline Haematocrit (HCT) & 107 & $16-57 \%$ & 36 & 7.2 & $17-61 \%$ & 38 & 7.6 \\
\hline $\begin{array}{l}\text { Red Blood Cells Count } \\
\text { (RBC) }\end{array}$ & 106 & $1.85-7.17 \times 10^{12} / \mathrm{L}$ & 4.37 & 0.81 & $1.81-7.23 \times 10^{12} / \mathrm{L}$ & 4.37 & 0.85 \\
\hline Platelets (PLT) & 107 & $29-584 \times 10^{9} / \mathrm{L}$ & 205 & 74 & $36-615 \times 10^{9} / \mathrm{L}$ & 260 & 84 \\
\hline $\begin{array}{l}\text { Mean Cell Volume } \\
\text { (MCV) }\end{array}$ & 107 & $54.2-100.8 \mathrm{fl}$ & 83 & 8.5 & $54.1-10.5 f l$ & 87.1 & 9.1 \\
\hline $\begin{array}{l}\text { Mean Cell Haemoglobin } \\
(\mathrm{MCH})\end{array}$ & 108 & $15.4-36.8 \mathrm{pg}$ & 27.2 & 3.2 & $15.4-37.9 \mathrm{pg}$ & 29 & 3.7 \\
\hline $\begin{array}{l}\text { Mean Cell Haemoglobin } \\
\text { Concentration (MCHC) }\end{array}$ & 108 & $28.4-37.2 \mathrm{~g} / \mathrm{dl}$ & 32.8 & 1.3 & $28.4-36.2 \mathrm{~g} / \mathrm{dL}$ & 33.4 & 1.5 \\
\hline $\begin{array}{l}\text { Red Cell Distribution } \\
\text { Width (RDW) }\end{array}$ & 108 & $11.8-31.2 \mathrm{fl}$ & 14.7 & 3.1 & $10.7-33.6 \mathrm{fl}$ & 14.1 & 3.4 \\
\hline $\begin{array}{l}\text { Mean Platelets Volume } \\
\text { (MPV) }\end{array}$ & 108 & $7.6-15.0 \mathrm{fl}$ & 9.4 & 1.1 & $4.9-11.7 \mathrm{fl}$ & 7.7 & 1.6 \\
\hline $\begin{array}{l}\text { Lymphocytes -Absolute } \\
\text { (Lym-Abs) }\end{array}$ & 108 & $0.7-6.0 \times 10^{9} / \mathrm{L}$ & 2.1 & 0.87 & $0.5-5.6 \times 10^{9} / \mathrm{L}$ & 2.2 & 0.88 \\
\hline $\begin{array}{l}\text { Lymphocytes -\% (Lym- } \\
\% \text { ) }\end{array}$ & 108 & $1.8-57.3 \%$ & 34.2 & 10.8 & $6.3-61.2 \%$ & 36.3 & 11 \\
\hline
\end{tabular}


Table 2: Summary of correlation and bias between CELL-DYN RUBY and Medonic M-Series Haematology Analysers

\begin{tabular}{|c|c|c|c|c|c|c|}
\hline Analyte & Sample size (N) & $\begin{array}{l}\text { Correlation Coeffi- } \\
\text { cient ( } \mathrm{r} \text { ) }\end{array}$ & $\begin{array}{l}\text { Cusum Test } \\
\text { p-value }\end{array}$ & $\begin{array}{l}\text { Differences data points } \\
\text { distribution pattern }\end{array}$ & $\begin{array}{c}\text { Bias }(95 \% \mathrm{CI}) \\
\text { based on Bland-Alt- } \\
\text { man's Plot }\end{array}$ & $\begin{array}{l}\text { Clinically Accept- } \\
\text { able Bias }\end{array}$ \\
\hline $\begin{array}{l}\text { Haemoglobin } \\
\text { (g/dL) }\end{array}$ & 107 & 0.99 & 0.57 & Skewed $(P=0.012)$ & $-0.84(-0.92,-0.76)$ & $+/-0.83$ \\
\hline $\begin{array}{l}\text { White Blood } \\
\text { Cell Count (x } \\
\left.10^{9} / \mathrm{L}\right)\end{array}$ & 106 & 0.985 & 0.72 & Normal $(P=0.243)$ & $-0.10(-0.16,-0.04)$ & $+/-0.33$ \\
\hline $\begin{array}{l}\text { Haematocrit } \\
(\%)\end{array}$ & 107 & 0.983 & 0.71 & Normal $(P=0.847)$ & $-1.71(-1.88,-1.54)$ & $+/-1.44$ \\
\hline $\begin{array}{l}\text { Red Blood } \\
\text { Cells Count (x } \\
\left.10^{12} / \mathrm{L}\right)\end{array}$ & 106 & 0.987 & 0.57 & Skewed $(P=0.043)$ & $0.00(-0.01,0.02)$ & $+/-0.18$ \\
\hline $\begin{array}{c}\text { Platelets (x } \\
\left.10^{9} / \mathrm{L}\right)\end{array}$ & 107 & 0.838 & 0.96 & Normal $(P=0.504)$ & $-55(-61.07,-49.22)$ & $+/-51$ \\
\hline $\begin{array}{c}\text { Mean Cell } \\
\text { Volume (fL) }\end{array}$ & 107 & 0.988 & 0.97 & Skewed $(P=0.005)$ & $-4.18(-4.37,-3.98)$ & $+/-2.5$ \\
\hline $\begin{array}{l}\text { Mean Cell Hae- } \\
\text { moglobin (pg) }\end{array}$ & 108 & 0.974 & 0.41 & Normal $(P=0.106)$ & $-1.89(-2.05,-1.72)$ & $+/-2.5$ \\
\hline $\begin{array}{c}\text { Mean Cell } \\
\text { Haemoglobin } \\
\text { Concentration } \\
\text { (g/dL) }\end{array}$ & 108 & 0.618 & 0.97 & Normal $(P=0.241)$ & $-0.68(-0.85,-0.51)$ & $+/-2.5$ \\
\hline $\begin{array}{l}\text { Red Cell Distri- } \\
\text { bution Width } \\
\qquad \%)\end{array}$ & 108 & 0.942 & 0.3 & Skewed $(P=0.005)$ & $0.65(0.53,0.78)$ & $+/-2.5$ \\
\hline $\begin{array}{l}\text { Mean Platelets } \\
\text { Volume (fL) }\end{array}$ & 108 & 0.687 & 0.55 & Normal $(P=0.764)$ & $1.65(1.37,1.84)$ & $+/-2.5$ \\
\hline $\begin{array}{l}\text { Lymphocytes } \\
\text { (Absolute) (x } \\
109 / \mathrm{L})\end{array}$ & 108 & 0.937 & 0.87 & Normal $(P=0.935)$ & $-0.17(-0.21,-0.13)$ & $+/-0.5$ \\
\hline $\begin{array}{l}\text { Lymphocytes } \\
(\%)\end{array}$ & 108 & 0.92 & 0.43 & Skewed $(P=0.005)$ & $-1.60(-2.11,-1.10)$ & $+/-0.5$ \\
\hline
\end{tabular}

Of the five WBCs components, comparison could only be done on lymphocytes since the Medonic-M can only estimate three differentials (Granulocytes, Lymphocytes and MID Cells), while the CD-Ruby can estimate all the five white blood cells components (Neutrophils, Lymphocytes, Monocytes, Basophils and Eosinophils).

All tested parameters showed strong positive correlations ( $\mathrm{r} \geq$ 0.60 ) and linear relationships between the measurements from the two analysers (Table 2).

The differences in measurements between the two methods for WBCs, HCT, MCH, MHCH, PLTs, MPV and LYM-ABS followed a normal distribution while those for HGB, RBCs, RDW, MCV and LYM-\% were skewed. Table 2 shows correlation coefficients and estimated bias (95\% CI) for each parameter with the corresponding clinically acceptable bias. Figure 1 shows the Bland-Altman plots for the normally distributed differences.

The difference in measurements between CD-Ruby and Medonic-M showed some bias for all the parameters evaluated except
RBC. The bias and their 95\% CI for HGB, WBCs, HCT, RBCs, PLTs, MCV, MCH, MCHC, RDW, MPV, LYM-Abs and LYM(\%) were $-0.84 \mathrm{~g} /$ dL (-0.92, -0.76), - $0.10 \times 109 / \mathrm{L}(-0.16,-0.04), 1.71 \%(-1.88,-1.54)$, $0.00 \times 1012 / \mathrm{L}(-0.01,0.02),-55 \times 109 / \mathrm{L}(-61.07,-49.22),-4.18 \mathrm{fL}$ $(-4.37,-3.98),-1.89 p g(-2.05,-1.72)-0.68 \mathrm{~g} / \mathrm{dL}(-0.85,-0.51), 0.65 \%$ $(0.53,0.78), 1.65 \mathrm{fL}(1.37,1.84),-0.17 \times 109 / \mathrm{L}(-0.21,-0.13)$ and $-1.6 \%(-2.11,-1.10)$ respectively. The bias of CD-Ruby in comparison to Medonic-M for WBC, RBC, RDW, MCH, MCHC, Lym-Abs, and MPV met the bias acceptance criteria based on the $95 \% \mathrm{CI}$; those of HGB and PLTS are acceptable but with lower accuracy, while those of HCT, MCV and Lym-\% fell outside acceptable bias limits. Figure 2 shows the bias interpretation in relation to clinical significance.

\section{Discussion}

The CD-Ruby and Medonic-M Haematology analysers showed positive strong correlations for all the red blood and white blood cells indices and platelets. All the bias between the two analysers were within clinically acceptable limits except those of HCT, MCV and LYM-\%. Of all the FBC parameters analysed by the two equip- 
ment, only HGB, RBC, WBC, and PLTS are actually measured while others are usually calculated from the measured parameters by the analysers software $[9,10]$. Interestingly, all the four measured parameters met the set clinically acceptable bias limits.
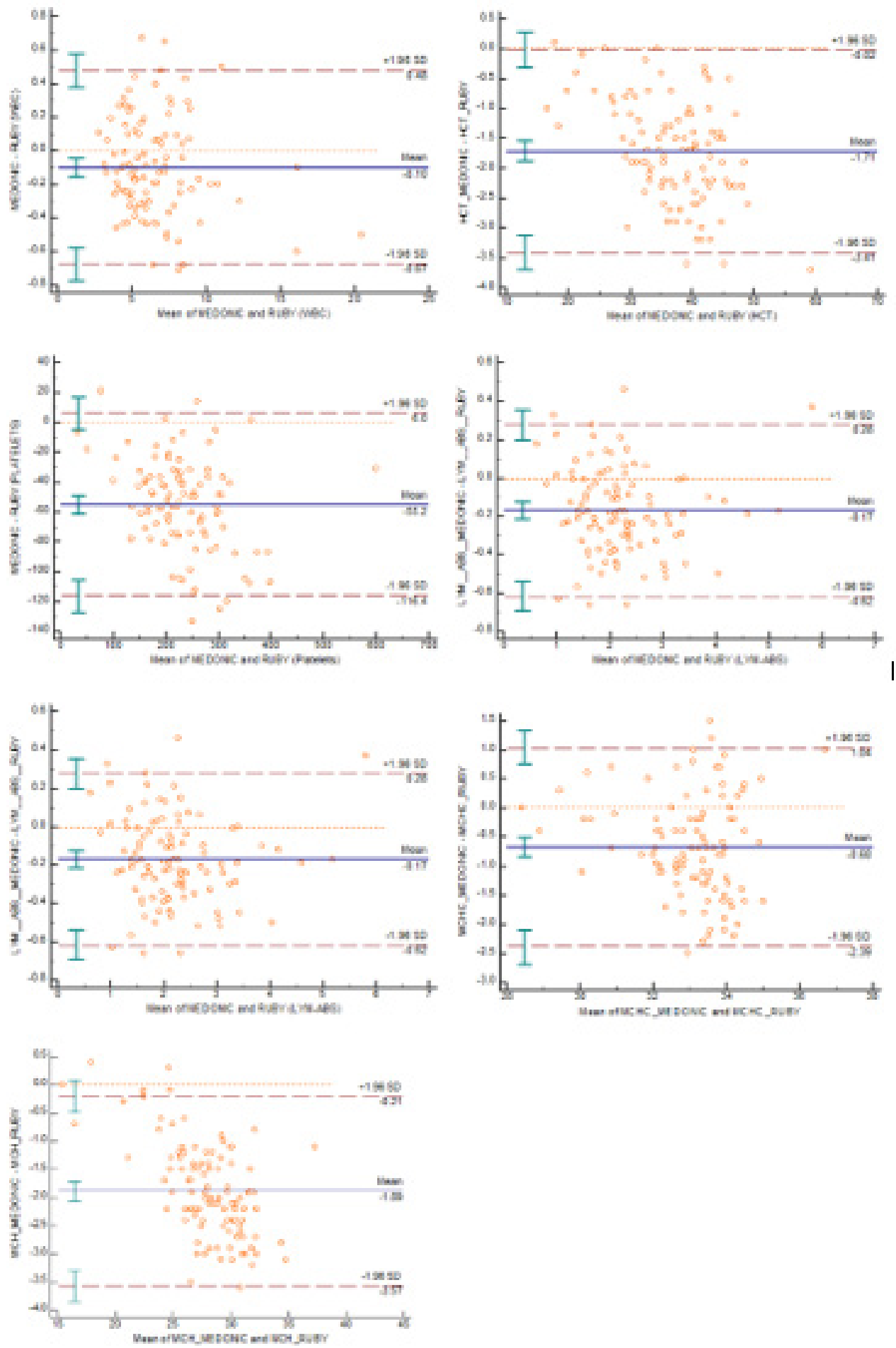

Figure 1: Bland-Altman's Plots for 7 parameters whose differences were normally distributed

White Blood Cells (WBC), Haemacrit (HCT), Platelets (PLTs), Mean Cell Haemaglobin (MCH), Mean Cell Haemoglobin Concentration (MCHC), Mean Platelets Volume (MPV), Lymphocytes - Absolutes (Lym-Abs) 


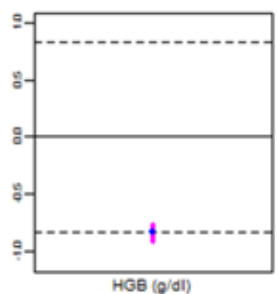

HGB (g/dI)

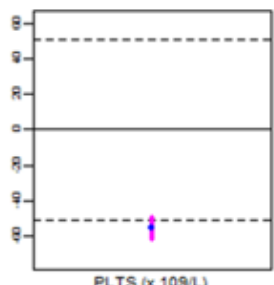

PLTS (x 109/L)

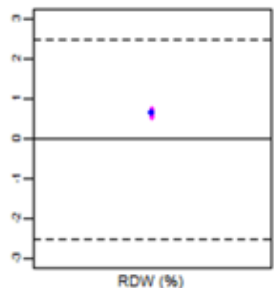

RDW (\$)

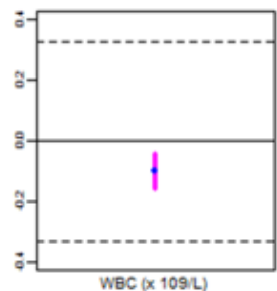

WBC $(\times 109 / 2)$

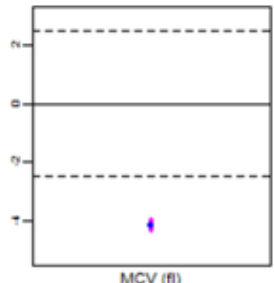

MCV (fi)

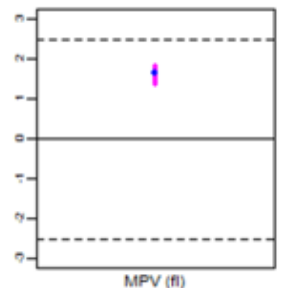

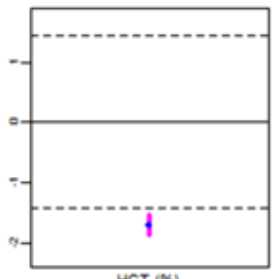

HCT (\%)

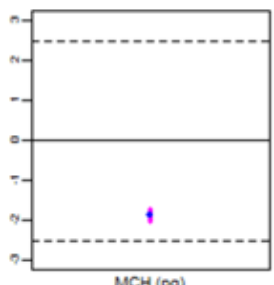

MCH (Pg)

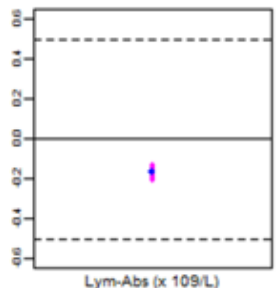

Lym-Abs (x 109/L)

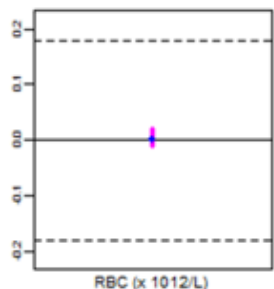

$\operatorname{RBC}(\times 1012 /)$

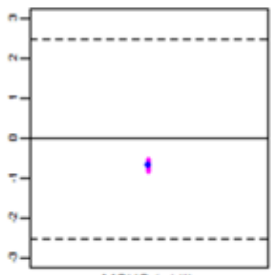

MCHC ( $/ / d$ d)

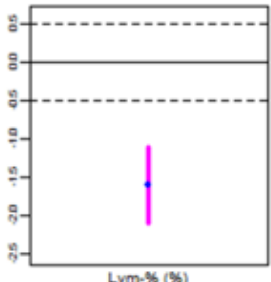

Figure 2: Bias and their $95 \% \mathrm{Cl}$ in Relation with Clinically Acceptable Limits for 12 hematological parameters Legends: Upper and lower clinically acceptable limits Bias Upper and Lower $95 \% \mathrm{Cl}$ of Bias

Haemoglobin (HGB), White Blood Cell Count (WBC), Hematocrit (HCT), Red Blood Cells Count (RBC), Platelets (PLT), Mean Cell Volume (MCV), Mean Cell Haemoglobin (MCH), Mean Cell Haemoglobin Concentration (MCHC), Red Cell Distribution Width (RDW), Mean Platelets Volume (MPV), Lymphocytes -Absolute (Lym-Abs), Lymphocytes -\% (Lym-\%).

All parameters showed negative bias, except RDW and MPV, suggesting that CD-Ruby is measuring higher than the Medonic-M and might therefore be more sensitive. This findings are in line with those reported by Leers et al [22] in their comparison of CD-Ruby with Sysmex XT-2000i. However, the rationale for the higher sensitivity of CD-Ruby as observed in our study, though not explored in detail here, might be attributed to its principles of measurement, which are the optical laser and flow cytometry, unlike the Medonic-M whose principle of measurement is impedance [23]. Further studies are required to substantiate this claim.

In line with the CLSI [7] interpretation of bias in relative to clinical significance, our findings showed that the bias for HGB, WBC, RBC, RDW, MCH, MCHC, Lym-Abs, PLTS and MPV met clinically acceptance criteria based on $95 \%$ CIs, we recommend that the CD-Ruby and Medonic-M are comparable and can be used interchangeably for the above mentioned parameters. On the other hand, the analysers were not comparable in our samples and may not be used interchangeably for HCT, MCV and LYM (\%) as their bias did not meet the clinically acceptable criteria; their results should be interpreted using the locally established equipment specific reference intervals [22].

The fact that the bias for MCV and HCT were out of clinically acceptable limits was not unexpected as MCV is a red blood cell index which is directly proportional to HCT, and it is calculated from HCT and RBC. The high bias observed for MCV in this present study also agrees with Leers et al who proposed that this might be due to different measuring principles of the two analysers [22]. Also the bias for LYM (\%) was unacceptable, whereas that of LYM-ABS was; both parameters are analyser-calculated lymphocytes values, expressed in percentage and absolute count respectively. This disparity might be due to variation in automated calculation software of the analysers.

Of great importance is that this study evaluated bias across low to high concentrations of medical decisions for all the parameters, and, given that FBC is a crucial laboratory investigation for diagnosis, case management and in clinical trials [3], replacement or backup laboratory methods for this test should have high level of agreement and any observed bias should be clinically acceptable. Further studies to assess comparability of various automated haematology analysers will be of great benefit.

Our study had a few limitations as it did not evaluate the flagging capacity or the operational efficiency comparability of the two analysers. In addition, we could not study comparisons of the WBCs differential since Medonic-M is a 3-parts differential haematology analyser, while CD-Ruby is 5-parts differential haematology analyser. 


\section{Conclusion}

This study has indicated that the Medonic-M and CD-Ruby Haematology analysers are generally comparable and can be used interchangeably for full blood count parameters except for HCT, MCV and Lym-\% which require results being interpreted using equipment specific reference intervals. The results of this study may help laboratories and clinics in selecting comparable methods for FBC. Moreover, the two study analysers have closed sampling facilities which make them safe for handling suspected infectious samples, as contact with personnel is minimal.

\section{Acknowledgements}

We acknowledge all the staff of Clinical laboratories at MRCG at the London School of Hygiene and Tropical Medicine for their supports during the study.

\section{Conflicts of Interest}

The authors have no competing interest. The authors declare that no fund or any gift was received to do this study.

\section{References}

1. Troussard, Sylviane V, Edouard C, Valérie B, Couaillac JP, et al. (2014) Full blood count normal reference values for adults in France. J Clin Pathol 67(4): 341-344.

2. Chisale MR, Kumwenda P, Ngwira, Baya, Chosamata, et al. (2015) A pilot study to determine the normal hematological indices for young Malawian adults in Blantyre, Malawi. Malawi Med J 27(3): 96-100.

3. Chavan P, Vivek B, Tiwari M, Umakant G, Sanjay P, (2016) Comparison of Complete Blood Count Parameters between Venous and Capillary Blood in Oncology Patients. J lab physicians 8(1): 65-66.

4. Fanshawe, José M, Philip J, Ann B, Brian S, et al. (2018) Frequencies and patterns of laboratory test requests from general practice: a service evaluation to inform point-of-care testing. J Clin Pathol 71(12): 10651071.

5. Johnson R, (2008) Assessment of Bias with Emphasis on Method Comparison. Clinical Biochem Rev 29(1): 37-42.

6. Theodorsson, Magnusson, Leito, (2014) Bias in clinical chemistry. Bioanalysis 6(21): 2855-2875.
7. CLSI, (2013) Measurement Procedure Comparison and Bias Estimation Using Patient Samples; Approved Guideline-Third Edition., in CLSI document EP09-A3. Clin Lab Standards Institute: Wayne, PA.

8. (2012) ISO 15189, Medical Laboratories-Requirements for quality and competence.

9. (2009) Boule Medical AB, Medonic Medonic M-Series User's Manual, B.M. AB, Editor. Sweden.

10. (2006) Abbott Laboratories, CELL-DYN Ruby System Operators' Manual 9140559B-August.

11. Medcalc, for Windows Version 17.6. MedCalc Software, Ostend, Belgium.

12. MINITAB INC (2017) Minitab, Inc: United States.

13. Tukey, John W, (1977) Exploratory Data Analysis.

14. Bland JM, Altman, (1986) Statistical methods for assessing agreement between two methods of clinical measurement. Lancet 1(8476): 307310 .

15. Mukaka MM, (2012) A guide to appropriate use of Correlation coefficient in medical research. Malawi Med J 24(3): 69-71.

16. Schober, Boer, Schwarte, (2018) Correlation Coefficients: Appropriate Use and Interpretation. Anesth Analg 126(5): 1763-1768.

17. Bilić Zulle L, (2011) Comparison of methods: Passing and Bablok regression. Biochemia Medica 21(1): 49-52.

18. Anderson, Darling, (1954) A test of goodness of fit. J Amer Statist Assoc 49(268): 765-769.

19. Giavarina D, (2015) Understanding Bland Altman analysis. Biochemia Med 25(2): 141-151.

20. (2019) C.f.M.a.M., Clinical Laboratory Improvement Amendments of 1988 (CLIA) Proficiency Testing Regulations Related to Analytes and Acceptable Performance. Department of Health and Human Services pp1536-1567 USA.

21. Leers, Goertz, Feller, Hoffmann, (2011) Performance evaluation of the Abbott CELL-DYN Ruby and the Sysmex XT-2000i haematology analysers. Int J Lab Hematol 33(1): 19-29.

22. Gresele P, Kleiman, Lopez, Page, (2017) Platelets in Thrombotic and Non-Thrombotic Disorders- Pathophysiology, Pharmacology and Therapeutics: An Update. Springer. 